\title{
Combined use of biochemical and volumetric biomarkers to assess the risk of conversion of mild cognitive impairment to Alzheimer's disease
}

\author{
Marta Nesteruk ${ }^{1}$, Tomasz Nesteruk ${ }^{2}$, Maria Styczyńska ${ }^{3}$, Monika Mandecka ${ }^{3}$, Anna Barczak ${ }^{3}$, Maria Barcikowska ${ }^{3}$ \\ ${ }^{1}$ Department of Neurology, Central Clinical Hospital of the Ministry of Interior, Warsaw, ${ }^{2}$ Department of Radiology, Central Clinical \\ Hospital of the Ministry of Interior, Warsaw, ${ }^{3}$ Department of Neurodegenerative Disorders, Mossakowski Medical Research Centre, \\ Polish Academy of Sciences, Warsaw, Poland
}

\begin{abstract}
Introduction: The aim of our study was to evaluate the usefulness of several biomarkers in predicting the conversion of mild cognitive impairment (MCI) to Alzheimer's disease (AD): $\beta$-amyloid and tau proteins in cerebrospinal fluid and the volumetric evaluation of brain structures including the hippocampus in magnetic resonance imaging (MRI). Material and methods: MRI of the brain with the volumetric assessment of hippocampus, entorhinal cortex, posterior cingulate gyrus, parahippocampal gyrus, superior, medial and inferior temporal gyri was performed in 40 patients diagnosed with mild cognitive impairment. Each patient had a lumbar puncture to evaluate $\beta$-amyloid and tau protein (total and phosphorylated) levels in the cerebrospinal fluid. The observation period was 2 years.

Results: Amongst 40 patients with MCl, 9 (22.5\%) converted to AD within 2 years of observation. Discriminant analysis was conducted and sensitivity for $\mathrm{MCl}$ conversion to $A D$ on the basis of volumetric measurements was $88.9 \%$ and specificity $90.3 \%$; on the basis of $\beta$-amyloid and total tau, sensitivity was $77.8 \%$ and specificity $83.9 \%$. The combined use of the results of volumetric measurements with the results of proteins in the cerebrospinal fluid did not increase the sensitivity (88.9\%) but increased specificity to $96.8 \%$ and the percentage of correct classification to $95 \%$.
\end{abstract}

Key words: mild cognitive impairment, conversion, biomarkers, volumetry, $\beta$-amyloid, Alzheimer's disease, cerebrospinal fluid, magnetic resonance imaging, hippocampus.

\section{Introduction}

Mild cognitive impairment $(\mathrm{MCl})$ was treated in the past as a transitional state between the physiological aging and dementia. Currently it is a separate diagnosis, although very heterogeneous. It requires clinical vigilance because of possibility of conversion to dementia, most often to Alzheimer's disease (AD), with an average of $7-15 \%$ per year. The moment of conversion is very important due to the possibility of therapeutic effects, which are most effective in the early stages of $A D$, while the recommended treatment of $\mathrm{MCl}$ does not exist. Criteria for diagnosis of AD (NIA/AA, 2011) [1] include not only the dementia phase but also the $\mathrm{MCl}$ phase and preclinical phase 
of Alzheimer's disease pathophysiological process, when pathological changes are present in the brain but the patient does not have any clinical symptoms. Such state can last for even twenty years.

Although "amyloid cascade hypothesis" has given rise to doubts [2], diagnostic criteria of $\mathrm{MCl}$ from 2011 indicate the important role of biomarkers [1]. Biomarkers can improve the prediction of $\mathrm{MCl}$ conversion to $A D$. Significant markers include markers of $\beta$-amyloid $(A \beta)$ deposition (decreased level of $A \beta_{1-42}$ in the cerebrospinal fluid (CSF) or positive amyloid imaging in PET) and markers of neuronal injury (increased levels of tau protein- total and/or phosphorylated in CSF or decreased glucose uptake in the temporal-parietal area in FDG-PET or reduced volume of hippocampus in magnetic resonance imaging MRI) [1]. Currently, these parameters are not used in clinical practice because of the lack of treatment of $\mathrm{MCl}$ due to AD. However, positive biomarkers increase the likelihood that the cognitive impairment can be caused by the pathophysiological process of $A D$ [9]. In such case the probability of $\mathrm{MCl}$ conversion to $\mathrm{AD}$ in the future is higher.

The aim of our study was to evaluate the usefulness of several biomarkers in predicting the conversion of $\mathrm{MCl}$ to $\mathrm{AD}$ : $\beta$-amyloid and tau proteins in the CSF and volumetric evaluation of different brain structures including the hippocampus in MRI.

\section{Material and methods}

The study population was 40 patients ( 22 women and $18 \mathrm{men}$ ), aged 50-80 years, with $\mathrm{MCl}$ diagnosed in the Alzheimer's Department (according to the diagnostic criteria from 2004; Winblad et al.) [16]. The Mini Mental State Examination (MMSE) [7], neurological and neuropsychological assessments (using standard neuropsychological tests) were performed; on CDR scale all patients received 0.5 [10]. Laboratory tests were taken to exclude other causes of cognitive impairment. Brain MRI was performed for all patients on a $1.5 \mathrm{~T}$ Toshiba apparatus to calculate volumes of selected structures (hippocampus, entorhinal cortex, posterior cingulate gyrus, parahippocampal gyrus, superior, medial, inferior temporal gyri and total intracranial volume) using FreeSurfer software. Each volume (hippocampus, entorhinal cortex, posterior cingulate gyrus, parahippocampal gyrus, superior, medial, inferior temporal gyri) was divided by the total intracranial volume to normalize results and to eliminate differences in the brain size (according to Whitwell) [15]. All volumes were multiplied by 1000 in order to facilitate comparison between them. Each patient had a lumbar puncture to evaluate $A \beta$ and tau protein (total and phosphorylated) in the cerebrospinal fluid. There was a 2-year observation period. During control visits, MMSE, neurological and neuropsychological examinations were performed to assess potential disease progression to AD. Alzheimer's disease was recognized on the basis of the diagnostic criteria NIA/AA, 2011 [1].

Patients diagnosed with conversion to AD had been treated with the acetylcholinesterase inhibitor. All patients have remained under the care of our Memory Disorders Outpatient Clinic and have had periodical follow-up visits.

Table I. Characteristics of patients in studied subgroups with regard to Alzheimer's disease biomarkers concentration in cerebrospinal fluid

\begin{tabular}{|lccc|}
\hline Variable & MCl whole sample & MCl stable & Converters \\
\hline$N$ & 40 & 31 & 9 \\
\hline Age & $63.17(9.56)$ & $61.26(8.61)$ & $69.78(10.23)$ \\
\hline MMSE & $27.50(1.73)$ & $27.58(1.79)$ & $27.22(1.56)$ \\
\hline Years of education & $13.95(2.88)$ & $14.13(2.74)$ & $13.33(3.43)$ \\
\hline A $\beta_{1-42}$ & $607.873(269.92)$ & $653.026(242.96)$ & $452.344(314.16)$ \\
\hline tTau & $299.776(196.64)$ & $269.355(166.12)$ & $404.561(262.82)$ \\
\hline pTau 181 & $45.480(19.94)$ & $43.145(19.03)$ & $53.522(22.08)$ \\
\hline A $\beta_{1-42} \leq 609.54$ & 20 & $13(41.9 \%)$ & $7(77.8 \%)$ \\
\hline tTau $\geq 277.02$ & 17 & $11(35.5 \%)$ & $6(66.7 \%)$ \\
\hline pTau 181 $\geq 55.08$ & 10 & $7(22.6 \%)$ & $3(33.3 \%)$ \\
\hline
\end{tabular}

Data presented as mean (standard deviation)

$A \beta_{1-42}$ - CSF amyloid beta 1-42 (pg/ml), tTau - CSF total tau (pg/ml), pTau 181-CSF hyperphosphorylated tau at threonine 181 (pg/ml) 
Table II. Descriptive statistics in each subgroup (normalized volumes were multiplied by 1000)

\begin{tabular}{|lcccccc|}
\hline \multirow{2}{*}{ Structure } & \multicolumn{2}{c}{ Non-converters $(n=31)$} & \multicolumn{2}{c|}{ Converters $(n=9)$} & \multicolumn{2}{c|}{ All $(n=40)$} \\
\cline { 2 - 7 } & Average & Standard deviation & Average & Standard deviation & Average & Standard deviation \\
\hline LH & 2.529 & 0.253 & 2.009 & 0.418 & 2.412 & 0.365 \\
\hline RH & 2.528 & 0.347 & 2.138 & 0.391 & 2.440 & 0.389 \\
\hline LERC & 0.596 & 0.138 & 0.447 & 0.128 & 0.562 & 0.149 \\
\hline RERC & 0.479 & 0.125 & 0.411 & 0.084 & 0.464 & 0.120 \\
\hline LPCG & 1.613 & 0.253 & 1.549 & 0.287 & 1.599 & 0.259 \\
\hline RPCG & 1.649 & 0.317 & 1.55 & 0.274 & 1.627 & 0.308 \\
\hline LPHG & 1.109 & 0.158 & 1.04 & 0.252 & 1.094 & 0.182 \\
\hline RPHG & 1.051 & 0.164 & 1.007 & 0.224 & 1.041 & 0.177 \\
\hline LITG & 5.794 & 0.777 & 5.167 & 1.144 & 5.653 & 0.896 \\
\hline LMTG & 5.432 & 0.591 & 5.216 & 0.991 & 5.383 & 0.692 \\
\hline LSTG & 6.111 & 0.814 & 5.567 & 1.168 & 5.988 & 0.918 \\
\hline RITG & 5.903 & 0.872 & 5.153 & 0.839 & 5.734 & 0.911 \\
\hline RMTG & 6.128 & 0.88 & 5.747 & 1.182 & 6.042 & 0.982 \\
\hline RSTG & 6.01 & 0.921 & 5.495 & 0.922 & 5.894 & 0.904 \\
\hline
\end{tabular}

$L H$ - left hippocampus, $R H$ - right hippocampus, $L E R C$ - left entorhinal cortex, $R E R C$ - right entorhinal cortex, $L P C G$ - left posterior cingulate gyrus, $R P C G$ - right posterior cingulate gyrus, LPHG - left parahippocampal gyrus, RPHG - right parahippocampal gyrus, LITG - left inferior temporal gyrus, LMTG - left medial temporal gyrus, LSTG - left superior temporal gyrus, RITG - right inferior temporal gyrus, RMTG - right medial temporal gyrus, RSTG - right superior temporal gyrus

Table III. Results of a Student's t-test

\begin{tabular}{|c|c|c|c|c|}
\hline & The value of $t$ statistics & Degree of freedom (df) & Significance (two-sided) & Average difference \\
\hline LH & -3.549 & 10 & 0.005 & -0.52 \\
\hline $\mathrm{RH}$ & -2.891 & 38 & 0.006 & -0.39 \\
\hline LERC & -3.022 & 14 & 0.009 & -0.15 \\
\hline RERC & -1.541 & 38 & 0.132 & -0.07 \\
\hline LPCG & -0.652 & 38 & 0.519 & -0.06 \\
\hline RPCG & -0.855 & 38 & 0.398 & -0.10 \\
\hline LPHG & -0.998 & 38 & 0.325 & -0.07 \\
\hline $\mathrm{RPHG}$ & -0.655 & 38 & 0.516 & -0.04 \\
\hline LITG & -1.910 & 38 & 0.064 & -0.63 \\
\hline LMTG & -0.820 & 38 & 0.417 & -0.22 \\
\hline LSTG & -1.596 & 38 & 0.119 & -0.54 \\
\hline RITG & -2.289 & 38 & 0.028 & -0.75 \\
\hline RMTG & -1.027 & 38 & 0.311 & -0.38 \\
\hline RSTG & -1.532 & 38 & 0.134 & -0.52 \\
\hline$\beta$-amyloid & -2.042 & 38 & 0.048 & -200.7 \\
\hline Total tau & 1.873 & 38 & 0.069 & 135.2 \\
\hline Phosphorylated tau & 1.390 & 38 & 0.172 & 10.4 \\
\hline
\end{tabular}

LH - left hippocampus, RH - right hippocampus, LERC - left entorhinal cortex, RERC - right entorhinal cortex, LPCG - left posterior cingulate gyrus, RPCG - right posterior cingulate gyrus, LPHG - left parahippocampal gyrus, RPHG - right parahippocampal gyrus, LITG - left inferior temporal gyrus, LMTG - left medial temporal gyrus, LSTG - left superior temporal gyrus, RITG - right inferior temporal gyrus, RMTG - right medial temporal gyrus, RSTG - right superior temporal gyrus. 


\section{Results}

Amongst 40 patients with $\mathrm{MCl}, 9(22.5 \%)$ converted to $A D$ within 2 years of observation (on average 9.2 months, SD 5.8). The study population was divided into two subgroups: subgroup 1: non-converters, who did not convert to $A D$ (31 patients) and subgroup 2: converters, who converted to $A D$ (9 patients). The characteristics of subgroups, including the results of CSF are shown in Table I, together with the cut-off points (established in our laboratory, described in our previous study [8]).

On the basis of our laboratory cut-offs the most corresponding was $A \beta_{1-42}$ value, which was positive for 7 of $9(77.8 \%)$ converters but for 13 of 31 nonconverters it was false positive. A positive value for total tau protein was obtained for 6 of $9(66.7 \%)$ converters and the value false positive for 11 patients with stable $\mathrm{MCl}$. The result of phosphorylated tau protein was positive only for $33 \%$ of converters. Table II presents descriptive statistics for all measured structures in MRI - average normalized values were multiplied by 1000 for easier data comparison.

Table III shows the results the Student's $t$-test significance of differences between subgroups for independent samples.

Statistically significant values were obtained for the left hippocampus, right hippocampus, left entorhinal cortex, right inferior temporal gyrus and $A \beta$ $(p \leq 0.05)$. Discriminant analysis model used all volumetric measurements and values of $A \beta$ and total tau to determine subgroup membership: converter or non-converter. Discriminant analysis was conducted in three steps: for volumetric measurements only, for $\mathrm{A} \beta$ and total tau (phosphorylated tau was excluded because of high $p$-value) and for volumetry and CSF biomarkers. Sensitivity for $\mathrm{MCl}$ conversion to $A D$ on the basis of volumetric measurements was $88.9 \%$ and specificity $90.3 \%$. On the basis of $A \beta$ and total tau sensitivity was $77.8 \%$ and specificity $83.9 \%$. The per- centage of correct classification using the results of the volumetric measurement was $90 \%$, and by using $A \beta$ and total tau $82.5 \%$. The results of the volumetric measurements together with results of the proteins in the CSF did not increase the sensitivity (88.9\%) but increased specificity to $96.8 \%$ and the percentage of correct classification to $95 \%$. Sensitivity, specificity and the percentage of correct classification for parameters which were statistically significant are presented in Table IV.

\section{Discussion}

The obtained results confirm that the use of volumetric assessment of selected brain structures and the assessment of $A \beta$ and tau protein in CSF can be useful in predicting the $\mathrm{MCl}$ progression to $A D$. However, the biggest limitation of our study was the small group of patients (40 persons), so the results are limited. Surprisingly, sensitivity for volumetric measurements was almost 90\%, whereas in our previous study (101 patients diagnosed with $\mathrm{MCl}$ ) we have obtained sensitivity of $64.7 \%$, specificity of $96.4 \%$ and classification rate of $91 \%$ (in this study 90\%) [11]. Similar results using volumetry were presented by Convit. His study group was also limited (46 patients); sensitivity of the prediction of conversion by using volume of hippocampus was $57 \%$ (in our study $66.7 \%$ ) and by using all measured volumes increased to $93 \%$ (in our study to $88.9 \%$ ), specificity was $97 \%$ (in our study $90.3 \%$ ) [3]. Taking into account individual volumetric measurements the results obtained in our previous study were confirmed, i.e. the highest sensitivity was for the hippocampus and then for the left entorhinal cortex [11]. Our results are contrary to the results presented by Dickerson (23 patients diagnosed with $\mathrm{MCl}$, observation period of 12-77 months) or Stoub (23 patients diagnosed with $\mathrm{MCl}$ and 35 from the control group, observation period was 5 years) in whose studies volume of

Table IV. Sensitivity, specificity and classification rate for single parameters

\begin{tabular}{|lccc|}
\hline & Sensitivity (\%) & Specificity (\%) & Correct classification rate (\%) \\
\hline LH & 66.7 & 77.4 & 75 \\
\hline RH & 66.7 & 74.2 & 72.5 \\
\hline LERC & 55.6 & 67.7 & 65 \\
\hline RITG & 55.6 & 64.5 & 62.5 \\
\hline A $\beta$ & 77.8 & 64.5 & 67.5 \\
\hline Total tau & 66.7 & 83.9 & 80 \\
\hline
\end{tabular}

LH - left hippocampus, $R H$ - right hippocampus, LERC - left entorhinal cortex, RITG - right inferior temporal gyrus 
entorhinal cortex was a better parameter than volume of hippocampus in predicting $\mathrm{MCl}$ conversion to $\mathrm{AD}[4,13]$. It should be noted that higher sensitivity compared to single volumetric measurements, was obtained for $A \beta$ (77.8\%), as in Egli's study, and as for total tau it was the same as for hippocampi (66.7\%) but total tau has had higher specificity compared with $A \beta$ and hippocampi which gives the best percentage of correct classification (conversion vs. no conversion) for total tau protein (80\%). Specificity increased after using a few parameters together [5].

Biomarkers were also studied in Ewers' study; the most sensitive parameter was volumetric measurement of left hippocampus and the highest percentage of correct classification was achieved by using the right entorhinal cortex volume. Sensitivity and specificity of prediction of $\mathrm{MCl}$ conversion to $\mathrm{AD}$ increased in the models using parameters of cerebrospinal fluid [6].

The study which used ADNI database [14] on 162 patients with diagnosed $\mathrm{MCl}$ showed superiority of the biomarkers from CSF in predicting the conversion of $\mathrm{MCl}$ to $\mathrm{AD}$ (sensitivity $76.4 \%$ vs. $65.4 \%$ ), the percentage of correct classification for both markers was the same (65.4\%) but increased (to 68.5\%) using both methods together (follow-up period of 36 months).

In Prestia's study the highest sensitivity was for $A \beta(79 \%)$ as a single biomarker, which was also confirmed in our work, with the highest specificity for the volumetric measurement of hippocampus (76\%). The study group consisted of 103 patients diagnosed with $\mathrm{MCl}$ (from two databases: ADNI and TOMC and follow-up period was $36 \pm 12$ months) [12].

The follow-up period for our study was 2 years and there is a possibility that in the coming years progression to $A D$ in subsequent patients can be observed, so the proportion of converters to nonconverters can change and sensitivity of used methods can also improve. The patients enrolled in our study met the $\mathrm{MCl}$ criteria [16]; conversion to AD was diagnosed in the patients who progressed to dementia and met criteria for probable $A D$ [9] but even in such a small group there is a probability of a mistake in diagnosis (other type of dementia for example FTD, DLB).

\section{Conclusions}

The above-mentioned biomarkers seem to be important parameters, in particular when biochemical biomarkers are used together with volumetric ones. Possibility of CSF analysis with $A \beta$ and tau protein assessment is nowadays easier. MRI is also widely available. Confirmation of effectiveness of the method requires the study and observation on a larger group of patients with diagnosed $\mathrm{MCl}$.

\section{Disclosure}

Authors report no conflict of interest.

\section{References}

1. Albert MS, DeKosky ST, Dickson D, Dubois B, Feldman HH, Fox NC, Gamst A, Holtzman DM, Jagust WJ, Petersen RC, Snyder PJ, Carrillo MC, Thies B, Phelps $\mathrm{CH}$. The diagnosis of mild cognitive impairment due to Alzheimer's disease: recommendations from the National Institute on Aging-Alzheimer's Association workgroups on diagnostic guidelines for Alzheimer's disease. Alzheimers Dement 2011; 7: 270-279.

2. Armstrong RA. A critical analysis of the 'amyloid cascade hypothesis'. Folia Neuropathol 2014; 52: 211-225.

3. Convit A, de Asis J, de Leon MJ, Tarshish CY, De Santi S, Rusinek H. Atrophy of the medial occipitotemporal, inferior, and middle temporal gyri in non-demented elderly predict decline to Alzheimer's disease. Neurobiol Aging 2000; 21: 19-26.

4. Dickerson BC, Goncharova I, Sullivan MP, Forchetti C, Wilson RS, Bennett DA, Beckett LA, deToledo-Morrell L. MRI-derived entorhinal and hippocampal atrophy in incipient and very mild Alzheimer's disease. Neurobiol Aging 2001; 22: 747-754.

5. Egli SC, Hirni DI, Taylor KI, Berres M, Regeniter A, Gass A, Monsch AU, Sollberger M. Varying strength of cognitive markers and biomarkers to predict conversion and cognitive decline in an early-stage-enriched mild cognitive impairment sample. J Alzheimers Dis 2015; 44: 625-633.

6. Ewers M, Walsh C, Trojanowski JQ, Shaw LM, Petersen RC, Jack CR Jr, Feldman HH, Bokde AL, Alexander GE, Scheltens P, Vellas B, Dubois B, Weiner M, Hampel H; North American Alzheimer's Disease Neuroimaging Initiative (ADNI). Prediction of conversion from mild cognitive impairment to Alzheimer's disease dementia based upon biomarkers and neuropsychological test performance. Neurobiol Aging 2012; 33: 1203-1214.

7. Folstein MF, Folstein SE, McHugh PR. "Mini-mental state". A practical method for grading the cognitive state of patients for the clinician. J Psychiatr Res 1975; 12: 189-189.

8. Mandecka M, Budziszewska M, Barczak A, Pepłońska B, Chodakowska-Żebrowska M, Filipek-Gliszczyńska A, Nesteruk M, Styczyńska M, Barcikowska M, Gabryelewicz T. Association between Cerebrospinal Fluid Biomarkers for Alzheimer's Disease: APOE Genotypes and Auditory Verbal Learning Task in Subjective Cognitive Decline, Mild Cognitive Impairment, and Alzheimer's Disease. J Alzheimers Dis 2016; 54: 157-168.

9. McKhann GM, Knopman DS, Chertkow H, Hyman BT, Jack CR Jr, Kawas CH, Klunk WE, Koroshetz WJ, Manly JJ, Mayeux R, Mohs RC, Morris JC, Rossor MN, Scheltens P, Carrillo MC, Thies B, Weintraub S, Phelps CH. The diagnosis of dementia due to Alzheimer's disease: recommendations from the National Insti- 
tute on Aging-Alzheimer's Association workgroups on diagnostic guidelines for Alzheimer's disease. Alzheimers Dement 2011; 7: 263-269.

10. Morris JC. The Clinical Dementia Rating (CDR): current version and scoring rules. Neurology 1993; 43: 2412-2414.

11. Nesteruk M, Nesteruk T, Styczyńska M, Barczak A, Mandecka M, Walecki J, Barcikowska-Kotowicz M. Predicting the conversion of mild cognitive impairment to Alzheimer's disease based on the volumetric measurements of the selected brain structures in magnetic resonance imaging. Neurol Neurochir Pol 2015; 49: 349-353.

12. Prestia A, Caroli A, Herholz K, Reiman E, Chen K, Jagust WJ, Frisoni GB. Translational Outpatient Memory Clinic Working Group; Alzheimer's Disease Neuroimaging Initiative. Diagnostic accuracy of markers for prodromal Alzheimer's disease in independent clinical series. Alzheimers Dement 2013; 9: 677 686.

13. Stoub TR, Bulgakova M, Leurgans S, Bennett DA, Fleischman D, Turner DA, deToledo-Morrell L. MRI predictors of risk of incident Alzheimer disease: a longitudinal study. Neurology 2005; 64: 1520-1524.

14. Westman E, Muehlboeck JS, Simmons A. Combining MRI and CSF measures for classification of Alzheimer's disease and prediction of mild cognitive impairment conversion. Neuroimage 2012; 62: 229-238.

15. Whitwell JL, Crum WR, Watt HC, Fox NC. Normalization of cerebral volumes by use of intracranial volume: implications for longitudinal quantitative MR imaging. AJNR Am I Neuroradiol 2001; 22: 1483-1489.

16. Winblad B, Palmer K, Kivipelto M, Jelic V, Fratiglioni L, Wahlund LO, Nordberg A, Bäckman L, Albert M, Almkvist O, Arai H, Basun H, Blennow K, de Leon M, DeCarli C, Erkinjuntti T, Giacobini E, Graff C, Hardy J, Jack C, Jorm A, Ritchie K, van Duijn C, Visser P, Petersen RC. Mild cognitive impairment - beyond controversies, towards a consensus: report of the International Working Group on Mild Cognitive Impairment. J Intern Med 2004; 256: 240-246. 